\title{
BMJ Open Evaluating comparative effectiveness of psychosocial interventions for persons receiving opioid agonist therapy for opioid use disorder: protocol for a systematic review
}

\author{
Danielle B Rice, ${ }^{1,2}$ Brian Hutton, ${ }^{1,3}$ Patricia Poulin, ${ }^{1,4,5}$ Beth A Sproule, ${ }^{6,7}$ \\ Dianna Wolfe, ${ }^{1}$ David Moher, ${ }^{1,3,8}$ Kednapa Thavorn, ${ }^{1,3}$ Gary Garber, ${ }^{1,3,9}$ \\ Sheena Taha, ${ }^{10}$ Amy Porath, ${ }^{10}$ Melanie Willows, ${ }^{11,12}$ Leila Esmaeilisaraji, ${ }^{1}$ \\ Fatemeh Yazdi, ${ }^{1}$ Beverley Shea, ${ }^{1}$ Becky Skidmore, ${ }^{1}$ Kimberly Corace ${ }^{1,12,13,14}$
}

To cite: Rice DB, Hutton B, Poulin P, et al. Evaluating comparative effectiveness of psychosocial interventions for persons receiving opioid agonist therapy for opioid use disorder: protocol for a systematic review. BMJ Open 2018;8:e023902. doi:10.1136/ bmjopen-2018-023902

- Prepublication history and additional material for this paper are available online. To view please visit the journal (http:// dx.doi.org/10.1136/bmjopen2018-023902).

$\mathrm{DBR}$ and $\mathrm{BH}$ contributed equally.

Received 30 April 2018

Revised 18 July 2018

Accepted 17 August 2018
Check for updates

(C) Author(s) (or their employer(s)) 2018. Re-use permitted under CC BY-NC. No commercial re-use. See rights and permissions. Published by BMJ.

For numbered affiliations see end of article.

Correspondence to

Dr Brian Hutton;

bhutton@ohri.ca

\section{ABSTRACT}

Introduction The opioid crisis has resulted in increasing rates of death caused by problematic opioid use. Current clinical guidelines recommend that individuals with persons with opioid use disorder (OUD) receive pharmacological (eg, opioid agonist therapy) and psychosocial (eg, cognitive behavioural therapy) therapy; however, the best combination of pharmacologic and psychosocial components is not known. Our objective of the planned study is to conduct a comprehensive systematic review to assess the relative benefits of psychosocial interventions as an adjunct to opioid agonist therapy among persons with OUD.

Methods and analysis A comprehensive search for randomised controlled trials published in English or French will be conducted from database inception to March 2018. The search will be conducted in MEDLINE and translated for Embase, PsycINF0 and the Cochrane Central Register of Controlled Trials. Two independent reviewers will screen, extract and assess risk of bias of eligible articles. Primary outcomes of interest will be treatment retention and opioid use (based on urinalysis results). Secondary outcomes will include self-reported opioid use, abstinence from illicit drugs, adherence to psychosocial therapy and opioid agonist therapy, risk for sexually transmitted disease, risk for blood borne pathogens, changes in mental health symptoms (eg, depression), measures of craving and changes in patients' quality of life and relevant adverse events. If sufficient data and adequate homogeneity exists, network meta-analyses (NMA) will be performed.

Ethics and dissemination This will be the first systematic review to incorporate NMA to compare psychosocial treatments used as an adjunct to opioid agonist therapy for OUD. Results of this review will inform clinical management of persons with OUD.

Trial registration number CRD42018090761.

\section{BACKGROUND}

Opioids are psychoactive analgesic drugs that can be prescribed for relief of acute or chronic pain, ${ }^{1}$ and their illicit use has risen to

\section{Strengths and limitations of this study}

- This will be the first systematic review incorporating network meta-analysis to compare the effectiveness of psychosocial treatments delivered in combination with opioid agonist therapy among people with opioid use disorder.

- Subgroup analyses are planned for specialty groups such as youth, pregnant women, indigenous people and people who are incarcerated.

- Variability of outcome measures may influence the ability to perform planned network meta-analyses, and strategies have been planned to address this challenge.

epidemic levels. ${ }^{2}$ Opioid addiction, overdose and poisonings have resulted in increasing rates of opioid-related death. ${ }^{3}$ In 2016, more than 2800 Canadians and 42000 Americans died from opioid poisoning. ${ }^{45}$ An estimated 11.5 million individuals in the USA used opioid prescriptions non-medically in the past year alone. ${ }^{2}$ In Europe, $84 \%$ of drug-related deaths in 2015 were related to opioid use, with heroin (often in combination with other drugs) contributing to more than half of drug-related deaths. ${ }^{6}$ Problematic opioid use is also prevalent in Asia, which accounts for approximately two-thirds of all opiate users.

Non-medical use of prescription opioids as well as the use of illicit opioids are of particular concern. In Europe, heroin use continues to account for the majority (approximately $80 \%$ ) of new opioid-related treatment. ${ }^{8}$ There has also been a dramatic rise in illicit drugs being contaminated with fentanyl in both North America and Europe. In 2016, 
there was a $281 \%$ increase in the number of deaths attributable to fentanyl within Canada as compared with the preceding year. ${ }^{59}$ These alarming trends have prompted recent international, national and provincial level actions including the development of the 2017 Canadian Guideline for Opioids for Chronic Non-Cancer Pain, and Canadian, American, WHO and European guidelines for opioid use disorder (OUD), ${ }^{30-12}$ and the encouragement of prescribing cautiously and in smaller quantities to decrease misuse and dependence. ${ }^{13}$

Non-medical and illicit use of opioids can result in individuals developing an OUD. Compared with healthy individuals without OUD, individuals with an OUD have increased psychosocial distress, healthcare utilisation, morbidity and mortality. ${ }^{14} 15$ The societal implications of OUD are also substantial, as the total programme spending on drugs used for OUD (ie, methadone and buprenorphine/naloxone) exceeded $\$ 135$ million in Canada in $2015 .^{16}$ In the USA, the total economic burden of opioid overdose, addiction and dependence was $\$ 78.5$ billion in $2013 .{ }^{17}$ Given the costly and significant implications of OUD on mortality, morbidity, healthcare utilisation and societal costs, the identification of best management strategies is essential.

Clinical guidelines currently recommend that individuals with OUD receive concurrent therapy consisting of pharmacological (eg, opioid agonist therapy (OAT)) and psychosocial (eg, cognitive behavioural therapy) components. ${ }^{918}$ However, the best combination of pharmacological and psychosocial components is not currently known. To address the question of optimal effectiveness of pharmacotherapy, specifically, OAT, a systematic review and network meta-analysis (NMA) was planned to compare the effectiveness of OATs for the treatment of OUD. ${ }^{19}$ This review, however, could not be completed as intended due to substantial heterogeneity among outcome measures. Of 60 included trials, there were 53 different outcomes that were measured in 77 unique ways, ${ }^{19} 20$ thereby greatly complicating the ability to perform cross-study comparisons and pursue formal meta-analyses to compare OATs. Given the extensive variability among outcome measures, the authors were only able to conduct analyses on one outcome-treatment retention-where diacetylmorphine-assisted therapy was found to be superior to other OATs. ${ }^{20}$ Currently, no analogous systematic review and NMA for psychosocial therapies as adjunctive treatment to OAT for managing OUD exist. A variety of approaches have been used to aid in OUD management, including cognitive-behavioural therapy, motivational interviewing, contingency management, supportive counselling and other strategies. ${ }^{21}{ }^{22}$ While a quantitative comparison of the efficacy of psychosocial therapy has not been conducted, psychological treatment is considered by many clinicians to be a vital element to sustained success in people receiving OAT given its key role in ensuring treatment retention and improved outcomes. ${ }^{323}$

To our knowledge, no systematic review has included an NMA comparing the efficacy of psychosocial interventions used with OAT for individuals with OUD. NMA has become a vital and broadly used approach to evidence synthesis in addressing research questions that involve the comparison of multiple therapies and wherein relevant direct and indirect data exist. ${ }^{24-26}$ Such an analysis will be informative for decision making in addressing and treating the rising rate of OUD. The current protocol outlines a planned systematic review and NMA to compare the relative benefits of different psychosocial therapies among people with OUD receiving OAT.

\section{METHODS}

The reporting of this protocol adheres to the Preferred Reporting Items for Systematic Review and Meta-Analysis Protocols statement. ${ }^{27}$ The checklist for reporting items can be found in online supplementary appendix 1 . The review will be performed in accordance with the methods described below with any subsequent amendments described in the final manuscript. The review has also been registered in PROSPERO (CRD42018090761).

\section{Search strategy to identify relevant studies}

Search strategies to identify relevant studies for inclusion in the systematic review were developed and tested through an iterative process by an experienced medical information specialist (BecS) in consultation with the review team. Separate searches were performed for reviews and primary studies. The MEDLINE strategies were peer reviewed by another senior information specialist prior to execution using the PRESS Checklist. ${ }^{28}$ Using the OVID platform, we searched Ovid MEDLINE, including Epub Ahead of Print and In-Process \& Other Non-Indexed Citations, PsycINFO and Embase Classic+Embase. We also searched the Cochrane Library on Wiley. The review searches were performed on 28 February 2018 and the primary study searches on 8 March 2018. Strategies used a combination of controlled vocabulary (eg, 'Opiate Substitution Treatment', 'Opioid-Related Disorders/dt (drug therapy)', 'Buprenorphine/tu (therapeutic use)') and keywords (eg, 'opioid maintenance', 'methadone substitution' and 'OATS'). Randomised controlled trial, non-randomised controlled trial and systematic review filters were applied as applicable (see online supplementary appendix 2 for full search strategy). Vocabulary and syntax were adjusted across databases. Conference abstracts prior to 2016 were removed from Embase and CENTRAL, and dissertation abstracts were removed from PsycINFO. The reference lists of identified trials and reviews will be searched to ensure no relevant studies are missed. The supplemental searches will be integrated into a Preferred Reporting Items for Systematic Reviews and Meta-Analyses (PRISMA) flow diagram. ${ }^{29} 30$

\section{Study eligibility criteria}

Population

Individuals receiving treatment pharmacological (OAT) and psychosocial interventions for opioid use will be of interest. Individuals diagnosed with OUD as defined by the Diagnostic and Statistical Manual of Mental Disorders 
(DSM-5) or diagnosed with opioid dependence as defined by the International Classification of Disease or DSM-IV will also be of interest. No restrictions will be made for age or specialty populations (eg, youth, pregnant women and people who are incarcerated). Based on the availability of data, such uniquely different populations will be analysed separately given potential sociodemographic and other differences. If not feasible, the appropriateness of regression-based adjustments to account for cross-study differences in the representation of such populations will be assessed.

\section{Interventions and comparators of interest}

Psychosocial interventions (delivered with OAT) will be of interest. Studies evaluating the benefits of psychosocial interventions alone will be excluded from the systematic review. Studies using control groups of either OAT alone or 'standard medical management' will be eligible given their high likelihood of serving as important sources of indirect evidence ${ }^{25}$ if NMAs are performed. Descriptions of the primary psychosocial interventions to be included based on existing research and recommendations are presented in online supplementary appendix 3 .

\section{Outcomes}

The coprimary outcomes of interest will be treatment retention and opioid use (including abstinence from opioids and opioid use based on urinalysis results). Retention can be measured in several ways including the use of a continuous value, such as the number of days a patient continued in an OAT programme. ${ }^{31}$ Retention can also be measured as a binary outcome, such as the proportion of patients who completed the planned number of therapy sessions, ${ }^{32}$ or the number of patients who received treatment for a predetermined minimum number of sessions. ${ }^{33}$ Secondary endpoints of interest will include self-reported opioid use, abstinence from illicit drug use, alcohol use, adherence to psychosocial therapy, adherence to OAT, HIV risk behaviours, changes in mental health symptoms (depression, anxiety and suicidality), measures of craving and changes in patients' quality of life, and adverse events (eg, increases in substance use). We will capture data for all reporting formats of outcomes considered (this will also provide valuable data for the planned future development of a core endpoints set for OUD); once data collection is complete, we will review outcome definitions and cross-study data availability to identify those endpoints that may be best suited for NMAs (those with insufficient data will be summarised in other ways).

\section{Study designs}

Study designs of interest will include randomised controlled trials. Only studies published in English and French will be included. Observational studies, casecontrol studies, case series and case reports will be excluded.

\section{Screening for eligible studies}

Citations obtained from the literature searches will be imported into Distiller SR Software (Evidence Partners; Ottawa, Canada), which will be used for all stages of study screening and data extraction. Citations will be screened independently by two reviewers (among DBR, LE and FY) based on title, keywords and abstract (level 1 screening) and full-text articles (level 2 screening). Level 1 will be performed using a liberal accelerated approach (ie, only one reviewer needed to include a citation, while two reviewers will be needed to exclude) and will be supported using text mining methods within Distiller SR. Level 1 citations deemed potentially relevant or lacking sufficient information to exclude will be carried forward to level 2, which will be performed by two reviewers independently. Disagreements at level 2 screening will be resolved by discussion. Where consensus is not achieved following discussion, a third independent team member will be consulted (BH or KC). Prior to conducting screening at level 1 and level 2, a small number of abstracts/full texts will be piloted to establish agreement and consistency among reviewers. Of studies that report on the same cohort (eg, updates of different follow-up durations), the most up-to-date information will be retained, and a note of the duplicate sample will be made. The process of literature selection will be reported using a flow diagram as recommended by the PRISMA statement. ${ }^{29} 30$

\section{Process of data collection}

Primary data collection of included studies will be performed by two reviewers using a standardised electronic data collection form in Distiller SR Software. Data gathered from included studies will include the patient population, intervention and comparator information, outcomes reported and study design. Study characteristics (authors, year of publication, journal and countries of data collection), patient characteristics (eligibility criteria and number per group), patient demographics (age, sex and race), type of opioid use (prescription and/or illicit), cited rationale for opioid use (eg, chronic pain), duration of opioid use, mode of use (intravenous vs oral), comorbidities or other unique demographic traits, interventions (with description, including numbers and duration of sessions, setting and therapist expertise, if described), treatment setting (eg, community, physician office and penitentiary) and outcomes as described above. All study characteristics will be summarised in tabular form to facilitate inspection and discussion with clinical experts in terms of study heterogeneity, grouping of interventions and other such topics required to inform analysis; these tables will also be included in the final report. Collected data will be verified by a second reviewer for accuracy, with disagreements being settled by discussion.

\section{Risk of bias (RoB) assessments of included studies}

RoB will be assessed for all studies included in the review using the Cochrane RoB tool. ${ }^{34}$ The Cochrane RoB tool evaluates seven domains (ie, random sequence generation, allocation concealment, blinding, missing outcome 
data, selective outcome reporting, attrition and 'other sources of bias').$^{34}$ RoB assessments will be conducted by two reviewers (among DBR, LE and FY), and disagreements will be resolved through discussion or by a third reviewer (BH or $\mathrm{KC}$ ). Results from RoB appraisals will be summarised in the review and reported in full on an itemby-item basis in an online supplementary appendix. They will also be considered when contextualising the results for each outcome.

\section{Approach to evidence synthesis for NMA}

For outcomes with sufficient data for analysis, we will first conduct traditional meta-analyses of all pairwise comparisons in the evidence networks. If feasible, NMAs will be performed subsequently. Initial exploration of potential clinical and methodological heterogeneity among the included studies will be conducted using tabular and graphical approaches that will be discussed by members of the research team. The assumption of transitivity will be evaluated by inspection of differences in patient eligibility criteria and pertinent patient demographics between studies, including comorbidities (eg, comorbid pain and mental health diagnoses). Methodological differences between studies that could influence outcome measurements will be noted. We will describe any concerns related to the transitivity assumption or methodological heterogeneity within the final report and consider statistical strategies to address any concerns.

Standard pairwise meta-analyses will be conducted by fitting random-effects models in Comprehensive Meta-Analysis V.3 software (Biostat; Englewood, New Jersey, USA) to generate summary estimates and to assess statistical heterogeneity across included studies. Summary estimates will be reported as mean differences, standardised mean differences or ORs as appropriate with corresponding $95 \%$ CIs. Statistical heterogeneity will be measured by the $\mathrm{I}^{2}$ statistic. $\mathrm{I}^{2}$ values of $50 \%$ or higher will be considered indicative of potentially important heterogeneity that will be explored using established methods such as subgroup analysis, meta-regression and/or exclusion of outlier studies. If necessary, similar approaches will be conducted in NMAs to address existing heterogeneity. Comparison adjusted funnel plots will be performed to assess publication bias if NMAs are performed.

Where feasible, NMAs will be carried out separately for each clinical outcome of interest. Approaches used for these analyses will follow existing recommendations for modelling of unadjusted and adjusted models as outlined by guidance from experts at the National Institute for Health and Care Excellence. ${ }^{35}{ }^{36}$ Both fixed-effects and random-effects models will be fit for each outcome, within a Bayesian framework. Totals of 50000 or more burn-in iterations and 50000 or more sampling iterations will be used for all NMAs, and model convergence will be assessed using Gelman Rubin diagnostics. ${ }^{36}$ The fit of a model will be assessed by comparing its posterior residual deviance with the number of unconstrained data points (ie, the number of intervention arms across all studies) for the analysis. Selection between different models will be based on deviance information criteria (DIC) for each competing model, with a difference of five or more points suggesting an important difference.

For networks where statistical heterogeneity is high or the number of single-study connections is high, random-effects models will be preferred. Between-treatment differences of continuous endpoints (eg, change in quality of life) will be analysed using a model for mean differences or standardised mean differences as appropriate dependent on the degree of studies providing data on different measurement scales. Findings from analyses of dichotomous outcomes (eg, adherence of a certain number of days and response to treatment) will be reported in terms of ORs. The assumption of consistency will be assessed by (1) fitting unrelated means models and comparing the DIC with the corresponding consistency models and (2) reviewing scatterplots of the residuals from these models. Probabilities of treatment superiority will be estimated using the Surface Under the Cumulative Ranking curve (SUCRA), and ranking of treatments will be estimated by median treatment rankings, with corresponding $95 \%$ credible intervals. All NMAs will be performed using WinBUGS software V.1.4.3 (MRC Biostatistics Unit).

\section{Subgroup and sensitivity analyses}

Primary analyses will be unadjusted; however, additional analyses to assess heterogeneity will be pursued if feasible based on data availability and network geometry considerations (eg, few single-study connections). Primary analyses will be conducted to compare psychosocial therapies at the treatment level, and if data allows for it, we will establish broader categories of interventions for comparison (eg, individual, family, couples and group) and conduct subgroup analyses. Subgroup and/or meta-regression will also be conducted for age (between 12-17 and 18-25 years), sex (per cent of women and men), duration of OUD, most common method of administration (oral vs intravenous), type of opioid use (per cent prescription vs illicit), per cent with physical health comorbidities, per cent with other substance/ alcohol use, extent of comorbid pain and per cent of patients with mental health comorbidities, where data are available.

\section{Reporting of review findings}

Both graphical and numeric display of findings will be presented. For each feasible outcome of interest, this will include network diagrams (to display the availability of evidence for all possible treatment comparisons), pairwise comparisons between interventions with 95\% credible intervals for all treatments in the network and SUCRA values and median treatment rankings. We will use the checklist of the PRISMA Statement for Network Meta-Analysis to ensure all findings are clearly reported. ${ }^{30}$ If NMA is not feasible, pairwise meta-analysis findings will be reported in tables with a narrative summary. 


\section{Patient and public involvement}

Patients were not involved in the design of this review; however, this work will inform the development of a core endpoints initiative. This initiative will involve multiple patient groups to gather perspectives on key outcomes for the development of future trials and to disseminate review findings.

\section{DISCUSSION}

Improving the treatment of OUD is a fundamental component of mitigating the current opioid crisis and reducing the increasing morbidity and mortality associated with OUD in North America. Certain OAT medications are recommended for treating OUD as they are cost-effective and clinically effective. ${ }^{37}$ Regardless of the form of OAT administered, guidelines for the clinical management of OUD recommend that psychosocial components be included, as this can improve treatment retention and outcomes. ${ }^{9}$ Despite this recommendation, only a small minority of individuals receiving OAT are also provided psychosocial treatment. ${ }^{3839}$ This may relate to a lack of comparative evidence for the various psychosocial interventions that can be applied in combination with OAT for individuals with OUD. To our knowledge, this will be the first NMA looking to compare the efficacy of psychosocial interventions delivered with OAT among individuals with OUD.

We anticipate certain challenges in the context of the current review. One limiting obstacle may be the existence of excessive between-study heterogeneity with regard to the outcomes measured across studies that may limit formal synthesis, as was found in a recent systematic review that compared OATs for patients with OUD. ${ }^{20}$ Despite this challenge, our systematic review and accompanying narrative summary will allow for the synthesis of studies to guide our understanding of the potential usefulness of psychosocial interventions applied as an adjunct to OAT for patients with OUD. This potential challenge motivates additional research that we will pursue in the second phase of our research wherein we will use established methods and collaborate with methodologists, physicians, clinicians and patients to develop a core outcomes set for OUD through Delphi surveys and interviews that include these stakeholder groups. Developing a core outcome set will inform and improve future research of OUD, both by enhancing the consistency of evaluations performed in research studies of OUD as well as improving the ability of researchers to both perform meta-analyses and derive cross-study comparisons to inform decision making.

\footnotetext{
Author affiliations

${ }^{1}$ Ottawa Hospital Research Institute, Ottawa, Ontario, Canada

${ }^{2}$ Department of Psychology, McGill University, Montreal, Quebec, Canada

${ }^{3}$ School of Epidemiology and Public Health, University of Ottawa, Ottawa, Ontario, Canada

${ }^{4}$ Faculties of Social Sciences and Medicine, University of Ottawa, Ottawa, Ontario, Canada

${ }^{5}$ Pain Clinic, Ottawa Hospital, Ottawa, Ontario, Canada
}

${ }^{6}$ Department of Pharmacy, Centre for Addiction and Mental Health, Toronto, Ontario, Canada

${ }^{7}$ Leslie Dan Faculty of Pharmacy and Department of Psychiatry, University of Toronto, Toronto, Ontario, Canada

${ }^{8}$ Centre for Journalology, Clinical Epidemiology Program, Ottawa Hospital Research Institute, Ottawa, Ontario, Canada

${ }^{9}$ Public Health Ontario, Toronto, Ontario, Canada

${ }^{10}$ Canadian Center on Substance Use and Addiction, Ottawa, Ontario, Canada

${ }^{11}$ Department of Family Medicine, Faculty of Medicine, University of Ottawa, Ottawa, Ontario, Canada

${ }^{12}$ Substance Use and Concurrent Disorders Program, The Royal Ottawa Mental Health Centre, Ottawa, Ontario, Canada

${ }^{13}$ Department of Psychiatry, Faculty of Medicine, University of Ottawa, Ottawa, Ontario, Canada

${ }^{14}$ Institute of Mental Health Research, University of Ottawa, Ottawa, Ontario, Canada

Acknowledgements We would like to thank Raymond Daniel (Assistant Information Specialist) for his role in providing support in database management and document procurement.

Contributors DBR, BH and $\mathrm{KC}$ drafted the protocol. BecS created and tested the search strategies to be used in the bibliographic databases. DM and BevS provided methodological input for the protocol and planned systematic review. DBR, KC, PP, GG, ST, AP, MW and BAS provided clinical expertise during the protocol development stage and will also provide such expertise throughout the duration of the review and during drafting of the final manuscript. DBR, KT, LE, FY and DW will be involved in screening, data extraction and quality assessment. DBR, KC and BH will be involved in all data analyses. $\mathrm{DBR}, \mathrm{KC}$ and $\mathrm{BH}$ will coordinate all aspects of the review. $\mathrm{BH}$ conceived of and is the guarantor of the review. All authors provided critical feedback to the protocol and approved the protocol.

Funding Funding for this work is being provided by the Canadian Institutes of Health Research (CIHR) grant number 397976. DBR is supported by a Vanier Canada Graduate CIHR Scholarship and received a student grant from the Psychology Foundation of Canada.

Disclaimer The funders had no role in the design of the planned study or preparation of this protocol.

Competing interests $\mathrm{BH}$ has previously received honoraria from Cornerstone Research Group for the provision of methodologic advice related to systematic reviews and meta-analysis.

Patient consent Not required.

Provenance and peer review Not commissioned; externally peer reviewed.

Open access This is an open access article distributed in accordance with the Creative Commons Attribution Non Commercial (CC BY-NC 4.0) license, which permits others to distribute, remix, adapt, build upon this work non-commercially, and license their derivative works on different terms, provided the original work is properly cited, appropriate credit is given, any changes made indicated, and the use is non-commercial. See: http://creativecommons.org/licenses/by-nc/4.0/.

\section{REFERENCES}

1. Franklin GM, Rahman EA, Turner JA, et al. Opioid use for chronic low back pain: a prospective, population-based study among injured workers in Washington state, 2002-2005. Clin J Pain 2009;25:743-51.

2. Centers for Disease Control and Prevention (CDC). Vital signs: overdoses of prescription opioid pain relievers---United States, 1999--2008. MMWR Morb Mortal Wkly Rep 2011;60:1487-92.

3. Bruneau J, Ahamad K, Goyer MÈ, et al. Management of opioid use disorders: a national clinical practice guideline. CMAJ 2018;190:E24 7-E257.

4. Canadian Institute for Health Information. Canadian centre on substance abuse. Hospitalizations and emergency department visits due to opioid poisoning in canada: Canadian Institute for Health Information, 2016.

5. Centers for Disease Control Prevention, 2017. Drug overdose death data 2017. https://www.cdc.gov/drugoverdose/data/statedeaths. html (accessed 4 Jan 2018).

6. European Monitoring Centre for Drugs and Drug Addiction (EMCDDA). European drug report 2018: trends and developments. Luxembourg: Publications Office of the European Union, 2018. (accessed 5 Jul 2018). 
7. United Nations Office on Drugs and Crime, 2015. World Drug Report 2015. http://www.unodc.org/documents/wdr2015/World_Drug_ Report_2015.pdf

8. European Monitoring Centre for Drugs and Drug Addiction (EMCDDA). European drug report 2017: trends and developments. Luxembourg: Publications Office of the European Union, 2017.

9. British Columbia Centre on Substance Use, British Columbia Ministry of Health, 2017. A guideline for the clinical management of opioid use disorder. http://www.bccsu.ca/wp-content/uploads/2017/06/BCOUD-Guidelines_June2017.pdf (accessed on 15 Jan 2018).

10. European Monitoring Centre for Drugs and Drug Addiction (EMCDDA), 2011. Guidelines for the treatment of drug dependence: a european perspective. http://www.emcdda.europa.eu/system/files/ publications/651/SI_treatment-guidelines-p3_315812.pdf (accessed $8 \mathrm{Jul}$ 2018)

11. Department of Mental Health, Substance Abuse, World Health Organization, International Narcotics Control Board, United Nations Office on Drugs and Crime, 2009. Guidelines for the psychosocially assisted pharmacological treatment of opioid dependence. http:// apps.who.int/iris/bitstream/handle/10665/43948/9789241547543_ eng.pdf;jsessionid=7CBE9320168C572DC76AE3F822237657? sequence $=1$ (Accessed on 08/Jul/2018).

12. Kampman K, Jarvis M. American Society of Addiction Medicine (ASAM) National practice guideline for the use of medications in the treatment of addiction involving opioid use. J Addict Med 2015;9:358-67.

13. The Ontario Drug Policy Research Network, 2016. Safety and use of opioids: a summary of ODPRN research on prescription opioid use in Ontario. http://odprn.ca/wp-content/uploads/2016/08/FINALODPRN-Opioids-Summary-Report-Aug-2016_compressed.pdf (accessed 15 Jan 2018)

14. Kolodny A, Courtwright DT, Hwang CS, et al. The prescription opioid and heroin crisis: a public health approach to an epidemic of addiction. Annu Rev Public Health 2015;36:559-74.

15. Grande LA, Thompson EC, Au MA, Ma A, et al. Problem drug-related behavior and discontinuation of opioids following the introduction of an opioid management program. J Am Board Fam Med 2016;29:718-26.

16. Canadian Institute for Health Information. Prescribed drug spending in Canada, 2016: a focus on public drug programs: Canadian Institute for Health Information, 2016.

17. Florence CS, Zhou C, Luo F, et al. The economic burden of prescription opioid overdose, abuse, and dependence in the United States, 2013. Med Care 2016;54:901-6.

18. National Collaborating Centre for Mental Health. Drug misuse: psychosocial interventions national clinical practice guideline number 51. London: National Institute for Health and Clinical Excellence, 2008.

19. Dennis BB, Naji L, Bawor M, et al. The effectiveness of opioid substitution treatments for patients with opioid dependence: a systematic review and multiple treatment comparison protocol. Syst Rev 2014;3:105.

20. Dennis B. Chronic pain: a red herring or risk factor in the management of patients receiving opioid substitution therapy: McMaster University, 2015. (accessed 4 Jan 2018).

21. Dugosh K, Abraham A, Seymour B, et al. A systematic review on the use of psychosocial interventions in conjunction with medications for the treatment of opioid addiction. J Addict Med 2016;10:93-103.

22. Smedslund G, Berg RC, Hammerstrøm KT, et al. Motivational interviewing for substance abuse. Cochrane Database Syst Rev 2011:CD008063.
23. Livingston JD, Adams E, Jordan M, et al. Primary care physicians' views about prescribing methadone to treat opioid use disorder. Subst Use Misuse 2018;53:1-10.

24. Catalá-López F, Tobías A, Cameron C, et al. Network meta-analysis for comparing treatment effects of multiple interventions: an introduction. Rheumatol Int 2014;34:1489-96.

25. Caldwell DM, Ades AE, Higgins JP. Simultaneous comparison of multiple treatments: combining direct and indirect evidence. BMJ 2005;331:897-900.

26. Salanti G. Indirect and mixed-treatment comparison, network, or multiple-treatments meta-analysis: many names, many benefits, many concerns for the next generation evidence synthesis tool. Res Synth Methods 2012;3:80-97.

27. Moher D, Shamseer L, Clarke M, et al. Preferred reporting items for systematic review and meta-analysis protocols (PRISMA-P) 2015 statement. Syst Rev 2015;4:1.

28. Sampson M, McGowan J, Cogo E, et al. An evidence-based practice guideline for the peer review of electronic search strategies. J Clin Epidemiol 2009;62:944-52.

29. Hser $\mathrm{Yl}$, Li J, Jiang $\mathrm{H}$, et al. Effects of a randomized contingency management intervention on opiate abstinence and retention in methadone maintenance treatment in China. Addiction 2011;106:1801-9.

30. Maas J, Barton G, Maskrey V, et al. Economic evaluation: a comparison of methadone versus buprenorphine for opiate substitution treatment. Drug Alcohol Depend 2013;133:494-501.

31. Chopra MP, Landes RD, Gatchalian KM, et al. Buprenorphine medication versus voucher contingencies in promoting abstinence from opioids and cocaine. Exp Clin Psychopharmacol 2009;17:226-36.

32. Moher D, Liberati A, Tetzlaff $\mathrm{J}$, et al. Preferred reporting items for systematic reviews and meta-analyses: the PRISMA statement. PLoS Medicine 2008;6

33. Hutton B, Salanti G, Caldwell DM, et al. The PRISMA extension statement for reporting of systematic reviews incorporating network meta-analyses of health care interventions: checklist and explanations. Ann Intern Med 2015;162:777-84.

34. Higgins JP, Altman DG, Gøtzsche PC, et al. The Cochrane Collaboration's tool for assessing risk of bias in randomised trials. BMJ 2011;343:d5928.

35. Dias S, Sutton A, Welton N, et al, 2012. NICE DSU technical support document 3: heterogeneity: subgroups, meta-regression, bias and bias-adjustment. http://www.nicedsu.org.uk/TSD3\%20Heterogeneity. final\%20report.08.05.12.pdf (accessed on 2 Feb 2018).

36. Dias S, Welton N, Sutton A, et al, 2011. NICE DSU technical support document 2: a generalised linear modelling framework for pairwise and network meta-analysis of randomised controlled trials. http:// wwwnicedsuorguk

37. American Society of Addiction Medicine, 2013. Advancing access to addiction medicine: implications for opioid addiction treatment. https://www.asam.org/docs/default-source/advocacy/aaam implications-for-opioid-addiction-treatment_final (accessed 2 Feb 2018).

38. Ministry of Health Long Term Care, 2016. Methadone treatment and services advisory committee: final report. http://health.gov.on.ca/en/ public/programs/drugs/ons/docs/methadone_advisory_committee_ report.pdf (accessed 2 Feb 2018).

39. Centre for Addiction and Mental Health, 2016. Prescription opioid policy framework. https://www.camh.ca/en/hospital/about_camh/ influencing_public_policy/Documents/CAMHopioidpolicyframework. pdf (accessed 7 Feb 2018). 